\title{
Soy intake in association with menopausal symptoms during the first 6 and 36 months after breast cancer diagnosis
}

\author{
Tsogzolmaa Dorjgochoo, \\ Vanderbilt Epidemiology Center, Department of Medicine, Vanderbilt University Medical Center, \\ 2525 West End Avenue, Suite 600, Nashville, TN 37203-1738, USA
}

Kai Gu,

Shanghai Institute of Preventive Medicine, 1380 Zhong Shan Road (W), Shanghai 200336, China

Ying Zheng,

Shanghai Institute of Preventive Medicine, 1380 Zhong Shan Road (W), Shanghai 200336, China

\begin{abstract}
Asha Kallianpur,
Vanderbilt Epidemiology Center, Department of Medicine, Vanderbilt University Medical Center, 2525 West End Avenue, Suite 600, Nashville, TN 37203-1738, USA
\end{abstract}

\section{Zhi Chen,}

Vanderbilt Epidemiology Center, Department of Medicine, Vanderbilt University Medical Center, 2525 West End Avenue, Suite 600, Nashville, TN 37203-1738, USA

\section{Wei Zheng,}

Vanderbilt Epidemiology Center, Department of Medicine, Vanderbilt University Medical Center, 2525 West End Avenue, Suite 600, Nashville, TN 37203-1738, USA

Wei Lu, and

Shanghai Institute of Preventive Medicine, 1380 Zhong Shan Road (W), Shanghai 200336, China

\section{Xiao Ou Shu}

Vanderbilt Epidemiology Center, Department of Medicine, Vanderbilt University Medical Center, 2525 West End Avenue, Suite 600, Nashville, TN 37203-1738, USA

Xiao Ou Shu: Xiao-ou.shu@vanderbilt.edu

\section{Abstract}

\begin{abstract}
It has been suggested that soy food and its components may relieve menopausal symptoms (MPS) including hot flashes, night sweats, and vaginal dryness in healthy women. However, little is known about the effect of soy food intake on MPS in women with breast cancer. We examined associations of occurrence of MPS with soy food intake in 4,842 Chinese women aged 20-75 years who had non-metastatic breast cancer and had not used hormone replacement therapy. MPS were assessed at 6 and 36 months after cancer diagnosis using a standardized questionnaire, and associations with soy food intake were evaluated in multivariate regression analyses. Daily soy food intake was assessed at 6 months postdiagnosis and over the first 36 months postdiagnosis using a validated food frequency questionnaire. The prevalence of MPS was 56\% at 6 months and $63 \%$ at 36 months postdiagnosis with the hotflash being the most common MPS ( 44-55\%). Hot flashes occurred mainly in premenopausal breast cancer patients who were in the highest quartile of isoflavone intake at 6 months post-diagnosis $(\mathrm{OR}=1.20,95 \% \mathrm{CI}$ : $0.98-1.59)$ compared with the lowest quartile. This association was stronger at 36 months postdiagnosis $(\mathrm{OR}=1.59,95 \% \mathrm{CI}$ :
\end{abstract}

Correspondence to: Xiao Ou Shu, Xiao-ou. shu@vanderbilt.edu.

Conflict of interest None. 
1.02-2.48). We found no significant associations for any MPS, night sweats, or vaginal dryness. Neither tamoxifen use nor BMI modified the association between MPS and isoflavone intake. There was no evidence that soy food consumption reduced MPS among breast cancer patients. High soy intake may increase the prevalence of hotflashes among pre-menopausal patients. Our study suggests that soy acts as an estrogen antagonist in breast cancer patients.

\section{Keywords}

Breast cancer; Menopausal symptoms; Soy isoflavones; Tamoxifen; BMI

\section{Introduction}

Compared with healthy women, women treated for breast cancer experience menopausal symptoms (MPS), including vasomotor symptoms (hot flashes and night sweats) and gynecologic symptoms (vaginal dryness), more often and more severely as a consequence of the sudden decrease in estrogen levels associated with systemic chemotherapy, radiation therapy, and treatment with anti-estrogens [1-3]. Most healthy women who suffer from MPS during the menopausal transition seek medical treatment [4] and benefit from the use of hormone replacement therapy (HRT) or steroids [5]. The use of HRT among breast cancer patients, however, is undesirable, because estrogen is a major risk factor for breast cancer development and progression [5,6]. Soy foods and their bioactive components, mainly isoflavones, have been considered as an alternative to HRT, because soy phytoestrogens have estrogenic activity [7]. Studies have shown that soy foods and soy products may, in fact, alleviate MPS in healthy women [8-10]. However, other studies, including a systematic review and meta-analysis of randomized trials of non-hormonal therapies for menopausal hot flashes [11] and a systematic review of phytoestrogens and MPS [12], did not confirm these beneficial effects. Both observational and intervention studies, mainly conducted in Western populations, have also investigated the therapeutic effects of soy foods and soy supplementation on MPS in breast cancer patients; most of these have failed to demonstrate significant benefit [13,14]. Furthermore, a possible increase in the severity of vasomotor symptoms was reported with 12 months of phytoestrogen supplementation in women with breast cancer who participated in the Women's Healthy Eating and Living (WHEL) study [15].

Tamoxifen, an antiestrogen drug, is a selective estrogen receptor modulator (SERM) commonly used to treat breast cancer [16] and has been shown to be associated with the occurrence of MPS, particularly in younger women [1,3]. It has also been suggested that soy isoflavones act as a SERM [17], however, the combined effect of tamoxifen and soyfood on the occurrence of MPS among breast cancer patients is unclear.

Using data from a population-based cohort study of breast cancer patients, we evaluated the association of soy food intake with the prevalence of MPS (any or specific, including hot flashes, night sweats, and vaginal dryness) and over the course of the post breast cancer diagnosis period ( 6 and 36 months). We also investigated whether this association was modified by estrogen-related factors (menopausal status, body mass index [BMI], and tamoxifen use).

\section{Methods}

\section{Study participants}

This study includes participants of the Shanghai Breast Cancer Survival Study (SBCSS), a large, population-based, longitudinal study of 5,042 women with primary breast cancer, 
aged 20-75 years in Shanghai, China. Detailed descriptions of study methods have been published elsewhere [3,18]. Briefly, incident breast cancer cases diagnosed between April 2002 and April 2006 were identified from the population-based Shanghai Cancer Registry and recruited into the study approximately 6 months after cancer diagnosis. Of the 6,299 eligible cases indentified, 5,042 participated in the study. Of the remaining cases, $757(12 \%)$ refused, $421(6.7 \%)$ could not be contacted, and 159 (2.5\%) did not participate for other miscellaneous reasons, such as ill health or communication problems. After the exclusion of 28 patients with stage IV breast cancer and 172 patients who used HRT, 4,842 cases remained for the present study. All women provided written, informed consent, and the institutional review boards of both participating institutions approved the study protocol.

\section{Data collection}

Study participants completed interviewer-administered surveys 6, 18, and 36 months after diagnosis and provided detailed information on demographic characteristics, disease history, reproductive history, diet, physical activity, lifestyle factors, use of complementary, and alternative medicine, and quality of life (QOL). Menopausal status was defined as the cessation of menstruation for 12 months or longer at 6 and 36 months postdiagnosis. History of chronic disease before breast cancer diagnosis was collected and quantified using the Charlson co-morbidity score [19] and the International Classification of Diseases, Ninth Revision, Clinical Modification (ICD-9-CM) [20]. Anthropometric measurements, including height, weight, waist circumference, and hip circumference, were taken for calculation of BMI $\left(\mathrm{kg} / \mathrm{m}^{2}\right)$ and waist-to-hip ratio (WHR). Clinical and pathology data, including information on initial breast cancer treatments (mastectomy, radiation therapy, chemotherapy, immunotherapy, and/or tamoxifen), TNM stage, and tumor hormone receptor (ER and PR) status were obtained by reviewing medical charts and pathology slides. Commonly used chemotherapeutic drugs included cyclophosphamide, adriamycin and 5fluorouracil, taxotere, epirubicin-adriamycin, navelbine, and immunotherapy (interferon, interleukin, and thymosin). Menopausal status, BMI, and tamoxifen use were evaluated at 6 months postdiagnosis and over the first 36 months postdiagnosis.

All 4,842 patients provided information on MPS at 6 months postdiagnosis, and 3,472 patients did so at 36 months postdiagnosis. Detailed information was collected on MPS by asking participants: "Have you ever experienced any of the following menopausal symptoms since having breast cancer: hot flashes, night sweats, or vaginal dryness since breast cancer diagnosis?" Those who answered "Yes" to one of these symptoms were considered patients having "any MPS". Participants were also asked for dates of onset, and, if applicable, date of resolution of each symptom. Almost all women who reported having MPS since their breast cancer diagnosis continued to experience MPS at 6 months postdiagnosis.

Habitual dietary intake was assessed for specific time windows: at 6 months postdiagnosis and over the first 36 months postdiagnosis. We used a validated food frequency questionnaire [21] that was designed to measure consumption of soy foods commonly consumed in Shanghai, including tofu, soy milk and fresh soy beans, and other soy products, as well as meat, fish, and cruciferous vegetables. Daily nutrient consumption, including soy protein and isoflavone intake, was estimated by multiplying the amount and frequency of each food consumed by the nutrient content of the food item based on the Chinese Food Composition Tables 2002 [22] and summing across all relevant foods. In addition, the weighted average (WA) of daily intake of soy isoflavones over the first 36 months postdiagnosis was calculated by summing the intakes reported at each survey using the formula: $\mathrm{WA}=\operatorname{sum}($ iso $1 \times 6$, iso $2 \times 12$, iso3 $\times 18) / \operatorname{sum}(6,12,18)$, where iso1, iso 2 , and iso3 are the daily intakes of isoflavones (mg/day) at 6,18 , and 36 months postdiagnosis and where 6,12 , and 18 are the number of months of isoflavone intake. 


\section{Statistical analysis}

The primary outcomes of this analysis were the prevalence of any MPS and each of the three most commonly reported MPS (hot flashes, night sweats, and vaginal dryness) among the study participants. Soy isoflavone intake was treated as an independent variable. The estimated intake of soy isoflavones was normally distributed. Statistical analyses were carried out for MPS in relation to isoflavone intake at 6 months post cancer diagnosis and in relation to the weighted isoflavone intake over the first 36 months post cancer diagnosis to examine whether the associations differed over time. Differences in sociodemographic, lifestyle, and clinical factors were compared among breast cancer patients at 6 and 36 months postdiagnosis using Pearson's chi-square $\left(\chi^{2}\right)$ test for categorical variables or a twosided $t$-test for continuous variables. We also compared the distributions of these factors between breast cancer patients with and without any MPS and adjusted for age at diagnosis (categories) using the Cochran-Mantel-Haenszel Statistics (CMH) for categorical variables or Analysis of Variance (ANOVA) for continuous variables. Unconditional logistic regression was used to estimate odds ratios (ORs) and 95\% confidence intervals (CIs) for the association between any MPS or each specific MPS and quartiles of soy isoflavone intake. All OR estimates were adjusted for age at breast cancer diagnosis (continuous), educational level (categories), parity ( $0,1,2$ or $\geq 3$ ), BMI (continuous), menopausal status, Charlson co-morbidity score $(0 / \geq 1)$, self-perceived QOL (poor, average, or good), vitamin supplement use (yes/no), total meat intake (continuous), TNM stage (0-I, II, and III), and use of tamoxifen, immunotherapy, and chemotherapy (yes/no). Tests for trend were performed by entering categorical variables of the exposures of interest as continuous parameters in the models. All statistical tests were based on two-sided probability and a significance (alpha) level of 0.05 . To evaluate any modifying effect of estrogen-related conditions on soy isoflavone intake, the analyses were further stratified by menopausal status (pre- vs. postmenopausal), obesity status (BMI $<27.5 \mathrm{vs.} \geq 27.5 \mathrm{~kg} / \mathrm{m}^{2}$, cut-offs for obesity in Asians) [23], and tamoxifen use (yes vs. no). Tests for interaction were performed using the log-likelihood test. Statistical analyses were performed using SAS software, version 9.2 (SAS Institute, Cary, NC).

\section{Results}

As expected, the bivariate analyses showed that smoking, alcohol consumption, and total meat intake at 36 months after cancer diagnosis decreased slightly from 6 months after cancer diagnosis, while exercise participation, vitamin supplement use, postmenopausal status, and tamoxifen use increased during the same period (Table 1). Menopausal symptoms (MPS) were common among breast cancer patients at 6 months postdiagnosis. The overall prevalence of any MPS was 56\%, and prevalence of hot flashes was $44 \%$, night sweats was $35 \%$, and vaginal dryness was $9 \%$. These rates increased at 36 months postdiagnosis to $63 \%$ for any MPS, 55\% for hot flashes, $28 \%$ for night sweats, and $14 \%$ for vaginal dryness (Table 3). Patients with MPS were younger than patients without MPS (mean age 51.4 vs. 56.4 years, $P<0.01$ ) and were more likely to be pre-menopausal, to have had fewer children, to have a history of chronic disease (Charlson co-morbidity score $\geq 1$ ), and to have higher total meat intake (Table 2). More patients with MPS had hormone receptor positive $(\mathrm{ER}+/ \mathrm{PR}+)$ breast cancer and received chemotherapy, immunotherapy, and/or tamoxifen than patients who reported no MPS $(P<0.05$ for all, except for chemotherapy). In addition, patients with MPS were less likely to report "good" overall quality of life (QOL) than patients without MPS $(17 \%$ vs. $20 \%$, respectively; $P<0.01)$. The differences between patients with and without any MPS at 36 months postdiagnosis were significant, except for meat intake and immunotherapy. Overall, the average (mean) daily intake of soy isoflavones was $45.3 \mathrm{mg}$ at 6 months postdiagnosis and $47.6 \mathrm{mg}$ over the first 36 months postdiagnosis (data not shown). The mean intake of soy isoflavones was slightly 
higher among patients with MPS compared with patients without MPS at 6 and 36 months postdiagnosis, however, the difference was not significant (Table 2).

The results of the multivariate regression analyses showed no statistically significant association between daily soy isoflavone intake and the occurrence of any MPS or of specific MPS (Table 3). However, the odds of having hot flashes increased and reached borderline significance for the highest quartile of isoflavone intake among premenopausal women at 6 months postdiagnosis ( $\mathrm{OR}=1.20,95 \%$ CI: 0.98-1.59). At the 36 months postdiagnosis assessment, the ORs for any MPS and hot flashes increased among patients who were in the highest quartile of weighted isoflavone intake (>62.86 mg/day) compared with patients in the lowest quartile of the intake $(\leq 27.28 \mathrm{mg} / \mathrm{day})$, OR $=1.48,95 \%$ CI: $0.94-$ 2.34 for any MPS and OR $=1.59,95 \%$ CI: $1.02-2.48$ for hot flashes. Tests for multiplicative interaction between isoflavone intake and menopausal status were marginally significant at 36 months postdiagnosis for any MPS $(P$ interaction $=0.07$ ), but not for specific MPS.

The associations between daily intake of isoflavones and MPS by tamoxifen use are presented in Table 4. Overall, there were no significant associations between soy isoflavone intake and any MPS or specific MPS for either tamoxifen users or non-users, although the ORs for any MPS, hot flashes, and vaginal dryness were slightly elevated among tamoxifen users in the highest quartile of isoflavone intake $(\mathrm{OR}=1.12,95 \%$ CI: $0.88-1.43$ for any MPS, OR $=1.14,95 \%$ CI: $0.90-1.45$ for hot flashes, and OR $=1.16,95 \%$ CI: $0.77-1.75$ for vaginal dryness). At 36 months postdiagnosis, more women had become tamoxifen users than at 6 months postdiagnosis, and a positive association between the highest quartile of isoflavone intake and MPS occurrence was seen among both tamoxifen users $(\mathrm{OR}=1.18$, 95\% CI: $0.98-1.52$ for any MPS and OR $=1.19,95 \%$ CI: $0.92-1.54$ for hot flashes) and non-tamoxifen users ( $\mathrm{OR}=1.21,95 \% \mathrm{CI}$ : $0.78-1.89$ for any MPS and $\mathrm{OR}=1.35,95 \% \mathrm{CI}$ : $0.86-2.12$ for hot flashes, $P$ interaction $>0.05$ ).

The association between isoflavone intake and MPS varied slightly by women's obesity status (BMI <27.5.0 vs. $\geq 27.5 \mathrm{~kg} / \mathrm{m}^{2}$ ) at both 6 and 36 months postdiagnosis, $P$ interaction $>0.05$ for all (Table 5).

\section{Discussion}

In this large prospective cohort study of breast cancer survivors in China, we found that MPS were highly prevalent among women with recently diagnosed breast cancer, which is similar to rates reported for women recently treated for breast cancer in Western countries [24-26]. We did not observe an overall significant association between any MPS and soy isoflavone intake or in the analyses stratified by obesity and tamoxifen use. However, there was some indication that the highest quartile of soy isoflavone intake may be associated with an increased prevalence of MPS, primarily hot flashes among premenopausal women.

There are several biological properties associated with soy isoflavones that may provide possible explanations for their association with MPS. Soy isoflavones bind to estrogen receptors and activate estrogen response genes [27], although the hormone-like effect of soy isoflavones is much weaker than endogenous estrogens such as 17-estradiol and estrone [17]. It has been postulated that isoflavones work as estrogen antagonists in an environment with high levels of estrogen, such as in premenopausal women, but act as estrogen agonists in an environment with low levels of estrogen, such as in postmenopausal women [28]. In laboratory studies, the administration of phytoestrogens, particularly soy isoflavones, weakened the estrogen inhibitory effects of tamoxifen in human estrogen-dependent breast tumor cell lines in vitro and in vivo $[29,30]$ and flaxseed enhanced the effect of tamoxifen in 
an ovariecto-mised mouse model with breast tumors [31,32], supporting the hypothesis that phytoestrogens exhibit dual action on hormone-dependent cancers [29].

Studies have revealed the estrogen-agonist effect of soy products and have reported beneficial effects of soy food and soy compounds on lowering MPS, particularly vasomotor symptoms (hot flashes and night sweats) among healthy perimenopausal and postmenopausal women [8,33-35]. However, an earlier systematic review of 25 randomized trials concluded that soy foods do not relieve hot flashes among perimenopausal and postmenopausal women [12]. The results of this review were based on various soy products including food, beverages, extracts in capsules or tablets, and powders, as well as red clover extracts [12]. On the other hand, in a recent systematic review of 23 randomized controlled trials evaluating the efficacy of soy food, particularly soy isoflavones in association with vasomotor symptoms, there was evidence for reduced prevalence or severity of hot flashes with use of isoflavone supplements for at least 12 weeks among perimenopausal and postmenopausal women [8]. Few intervention studies have evaluated the effect of soy food and its components on MPS among breast cancer patients, and the results have been inconclusive or even contradictory [13-15,36]. In the Women's Healthy Eating and Living (WHEL) study, daily intake of soybeans and soy-containing foods was not associated with severity of vasomotor symptoms at baseline, while phyto-estrogen supplementation was significantly associated with an increased risk for moderate/severe vasomotor symptoms among women with breast cancer (excluding stage IV cases) after a 12-month dietary intervention [15]. However, in the WHEL Study, soybeans and selected soy-containing foods were examined based on use versus non-use, and the amounts of soy and its components were not quantified. Two trials designed for evaluation of short term soy or isoflavone supplements (from 50 to $114 \mathrm{mg} /$ day isoflavones for 12 weeks) for treatment of MPS in premenopausal or post-menopausal breast cancer survivors found no alleviation of vasomotor symptoms among premenopausal or postmenopausal women with breast cancer $[14,36]$.

There are several limitations of the present study that should be noted. Occurrence of MPS was based on self-reports and may have been affected by recall bias or influenced by comorbidity and overall QOL. However, the misclassification in the assessment of MPS is likely to be independent of soy food intake. We cannot exclude the possibility of nondifferential misclassification that results in an underestimation of the true association. Although we carefully adjusted for a wide range of covariates in the analyses, potential residual confounding effects cannot be completely ruled out. We only collected information on MPS based on occurrence or non-occurrence (yes vs. no), thus, we were unable to investigate the effect of soy intake on the severity of MPS.

The present study also has several noteworthy strengths. This study is the largest and only population-based study that has investigated the dose-response effect of soy food consumption on the occurrence of MPS among breast cancer patients. A well-validated dietary questionnaire was used to assess daily soy food intake in this study. The high response rate and availability of information on a wide range of potential confounders reduced bias in assessing the association of soy food intake with MPS. The high level of intake, as well as the wide range of soy foods consumed in our study population, increased the statistical power of the study.

In summary, we found no evidence that soy food consumption alleviates MPS among breast cancer patients. Instead, we found that high soy intake was related to possible higher prevalence of hot flashes in premenopausal breast cancer patients. Our study suggests that soy isoflavones may act as estrogen antagonists in breast cancer patients. We have previously reported that moderate soy food intake was associated with reduced mortality and 
recurrence among breast cancer patients [21]. The benefit of soy on mortality and recurrence appears to reach its peak at moderate levels of soy intake (36.6-62.7 mg/day). Further increases in intake did not confer greater benefit. Given that MPS have a significant negative impact on the QOL of breast cancer survivors [26,37], our study results suggest that women with breast cancer consume a moderate amount soy food to achieve its survival benefits, while avoiding its negative influences on MPS.

\section{Acknowledgments}

We are grateful to the research staff of the Shanghai Breast Cancer Survival Study in Shanghai, China for implementing the study and to all of the women who participated in the SBCSS. We also thank Dr. Fan Jin for her contributions to the field operations and Bethanie Hull and Rod Jones for their technical assistance in the preparation of this manuscript. Funding for this study was provided by the U.S. Department of Defense Breast Cancer Research Program (DAMD 17-02-1-0607) and the National Cancer Institute (R01 CA118229).

\section{References}

1. Carpenter JS, Andrykowski MA, Cordova M, et al. Hot flashes in postmenopausal women treated for breast carcinoma: prevalence, severity, correlates, management, and relation to quality of life. Cancer. 1998; 82:1682-1691. [PubMed: 9576289]

2. Crandall C, Petersen L, Ganz PA, Greendale GA. Association of breast cancer and its therapy with menopause-related symptoms. Menopause. 2004; 11:519-530. [PubMed: 15356404]

3. Dorjgochoo T, Gu K, Kallianpur A, et al. Menopausal symptoms among breast cancer patients 6 months after diagnosis: a report from the Shanghai Breast Cancer Survival Study. Menopause. 2009; 16:1205-1212. [PubMed: 19590459]

4. Freeman EW, Sherif K. Prevalence of hot flushes and night sweats around the world: a systematic review. Climacteric. 2007; 10:197-214. [PubMed: 17487647]

5. NIH Consens State Sci Statements; NIH State-of-the-Science Conference Statement on management of menopause-related symptoms; 2005. p. 1-38.

6. Dietel M. Hormone replacement therapy (HRT), breast cancer and tumor pathology. Maturitas. 2010; 65:183-189. [PubMed: 20005648]

7. Cederroth CR, Nef S. Soy, phytoestrogens and metabolism: a review. Mol Cell Endocrinol. 2009; 304:30-42. [PubMed: 19433245]

8. Jacobs A, Wegewitz U, Sommerfeld C, Grossklaus R, Lampen A. Efficacy of isoflavones in relieving vasomotor menopausal symptoms - a systematic review. Mol Nutr Food Res. 2009; 53:1084-1097. [PubMed: 19653225]

9. Lethaby AE, Brown J, Marjoribanks J, Kronenberg F, Roberts H, Eden J. Phytoestrogens for vasomotor menopausal symptoms. Cochrane Database Syst Rev. 2007:CD001395. [PubMed: 17943751]

10. Nagata C, Takatsuka N, Kawakami N, Shimizu H. Soy product intake and hot flashes in Japanese women: results from a community-based prospective study. Am J Epidemiol. 2001; 153:790-793. [PubMed: 11296152]

11. Nelson HD, Vesco KK, Haney E, et al. Nonhormonal therapies for menopausal hot flashes: systematic review and meta-analysis. JAMA. 2006; 295:2057-2071. [PubMed: 16670414]

12. Krebs EE, Ensrud KE, MacDonald R, Wilt TJ. Phytoes-trogens for treatment of menopausal symptoms: a systematic review. Obstet Gynecol. 2004; 104:824-836. [PubMed: 15458907]

13. MacGregor CA, Canney PA, Patterson G, McDonald R, Paul J. A randomised double-blind controlled trial of oral soy supplements versus placebo for treatment of menopausal symptoms in patients with early breast cancer. Eur J Cancer. 2005; 41:708-714. [PubMed: 15763646]

14. Nikander E, Kilkkinen A, Metsa-Heikkila M, et al. A randomized placebo-controlled crossover trial with phytoestro-gens in treatment of menopause in breast cancer patients. Obstet Gynecol. 2003; 101:1213-1220. [PubMed: 12798527]

15. Gold EB, Flatt SW, Pierce JP, et al. Dietary factors and vasomotor symptoms in breast cancer survivors: the WHEL Study. Menopause. 2006; 13:423-433. [PubMed: 16735939] 
16. Howell SJ, Johnston SR, Howell A. The use of selective estrogen receptor modulators and selective estrogen receptor down-regulators in breast cancer. Best Pract Res Clin Endocrinol Metab. 2004; 18:47-66. [PubMed: 14687597]

17. Barnes S. The biochemistry, chemistry and physiology of the isoflavones in soybeans and their food products. Lymphatic Res Biol. 2010; 8:89-98.

18. Shu XO, Zheng Y, Cai H, et al. Soy food intake and breast cancer survival. JAMA. 2009; 302:2437-2443. [PubMed: 19996398]

19. Charlson ME, Pompei P, Ales KL, MacKenzie CR. A new method of classifying prognostic comorbidity in longitudinal studies: development and validation. J Chronic Dis. 1987; 40:373383. [PubMed: 3558716]

20. Department of Health and Human Services. The international classification of diseases. US Government Printing Office; Washington, DC: 1998. 9th Rev. edn, ICD-9-CM

21. Shu XO, Yang G, Jin F, et al. Validity and reproducibility of the food frequency questionnaire used in the Shanghai Women's Health Study. Eur J Clin Nutr. 2004; 58:17-23. [PubMed: 14679362]

22. Yang, Y.; Wang, G.; Pan, X. Chinese Food Composition Tables 2002. Chinese Centre for Disease Control and Prevention; Beijing: 2002.

23. WHO Expert Consultation. Appropriate body-mass index for Asian populations and its implications for policy and intervention strategies. Lancet. 2004; 363:157-163. [PubMed: 14726171]

24. Canney PA, Hatton MQ. The prevalence of menopausal symptoms in patients treated for breast cancer. Clin Oncol (R Coll Radiol). 1994; 6:297-299. [PubMed: 7826921]

25. Ganz PA. Menopause and breast cancer: symptoms, late effects, and their management. Semin Oncol. 2001; 28:274-283. [PubMed: 11402437]

26. Gupta P, Sturdee DW, Palin SL, et al. Menopausal symptoms in women treated for breast cancer: the prevalence and severity of symptoms and their perceived effects on quality of life. Climacteric. 2006; 9:49-58. [PubMed: 16428125]

27. Penttinen-Damdimopoulou PE, Power KA, Hurmerinta TT, Nu-rmi T, van der Saag PT, Makela SI. Dietary sources of lignans and isoflavones modulate responses to estradiol in estrogen reporter mice. Mol Nutr Food Res. 2009; 53:996-1006. [PubMed: 19603405]

28. Hwang CS, Kwak HS, Lim HJ, et al. Isoflavone metabolites and their in vitro dual functions: they can act as an estrogenic agonist or antagonist depending on the estrogen concentration. J Steroid Biochem Mol Biol. 2006; 101:246-253. [PubMed: 16965913]

29. Helferich WG, Andrade JE, Hoagland MS. Phytoestrogens and breast cancer: a complex story. Inflammopharmacol. 2008; 16:219-226.

30. Tonetti DA, Zhang Y, Zhao H, Lim SB, Constantinou AI. The effect of the phytoestrogens genistein, daidzein, and equol on the growth of tamoxifen-resistant T47D/PKC alpha. Nutr Cancer. 2007; 58:222-229. [PubMed: 17640169]

31. Chen J, Hui E, Ip T, Thompson LU. Dietary flaxseed enhances the inhibitory effect of tamoxifen on the growth of estrogen-dependent human breast cancer (mcf-7) in nude mice. Clin Cancer Res. 2004; 10:7703-7711. [PubMed: 15570004]

32. Chen J, Power KA, Mann J, Cheng A, Thompson LU. Flaxseed alone or in combination with tamoxifen inhibits MCF-7 breast tumor growth in ovariectomized athymic mice with high circulating levels of estrogen. Exp Biol Med (Maywood). 2007; 232:1071-1080. [PubMed: 17720953]

33. Messina M, Hughes C. Efficacy of soyfoods and soybean isoflavone supplements for alleviating menopausal symptoms is positively related to initial hot flush frequency. J Med Food. 2003; 6:111. [PubMed: 12804015]

34. Umland EM. Treatment strategies for reducing the burden of menopause-associated vasomotor symptoms. J Manag Care Pharm. 2008; 14:14-19. [PubMed: 18439062]

35. Kronenberg F, Fugh-Berman A. Complementary and alternative medicine for menopausal symptoms: a review of randomized, controlled trials. Ann Intern Med. 2002; 137:805-813. [PubMed: 12435217] 
36. Van Patten CL, Olivotto IA, Chambers GK, et al. Effect of soy phytoestrogens on hot flashes in postmenopausal women with breast cancer: a randomized, controlled clinical trial. J Clin Oncol. 2002; 20:1449-1455. [PubMed: 11896091]

37. Dorjgochoo T, Kallianpur A, Zheng Y, et al. Impact of menopausal symptoms on quality of life 6 months after systemic breast cancer treatment: results from the Shanghai Breast Cancer Survival Study. Breast Cancer Res Treat. 2010; 119:725-735. [PubMed: 19543973] 


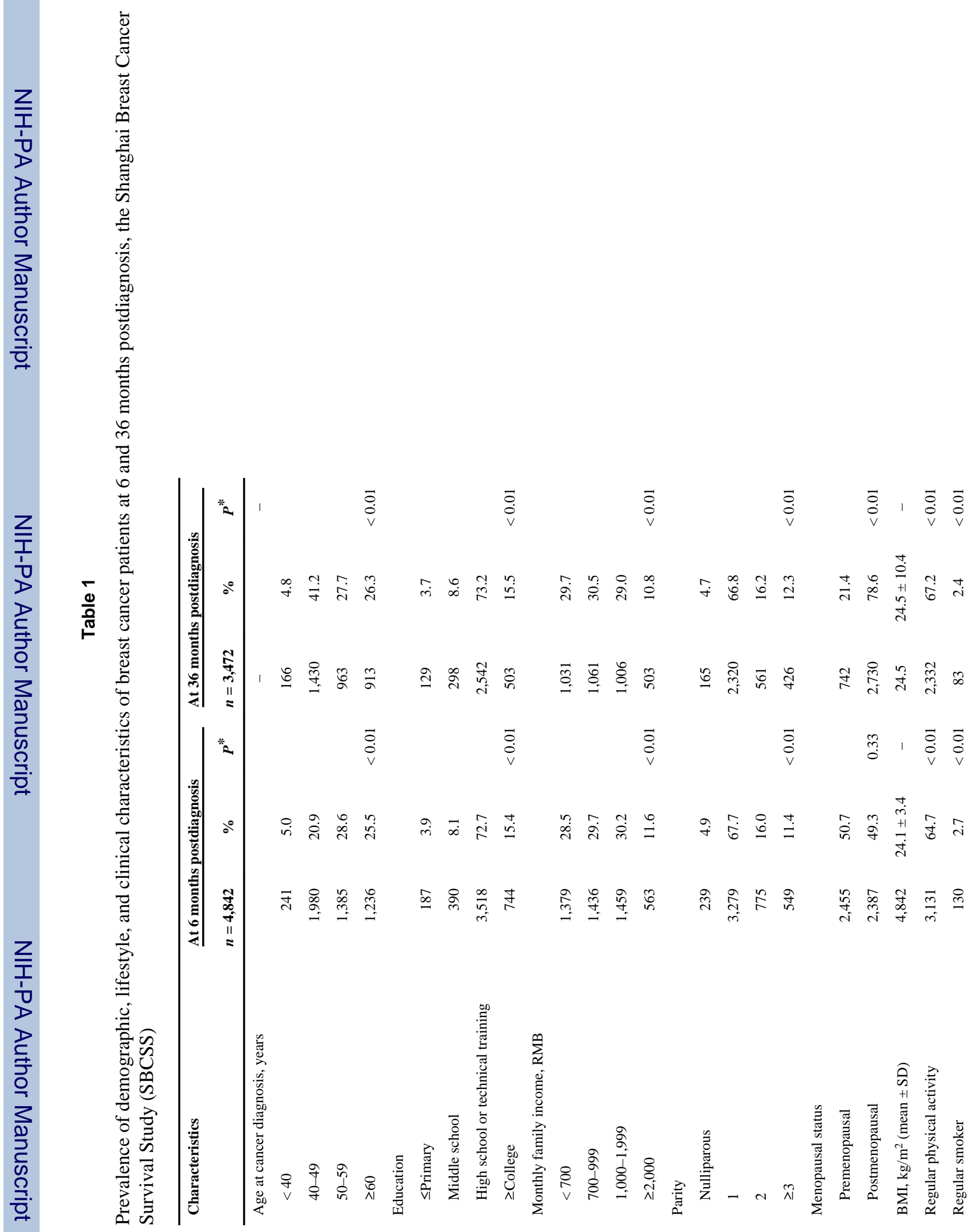




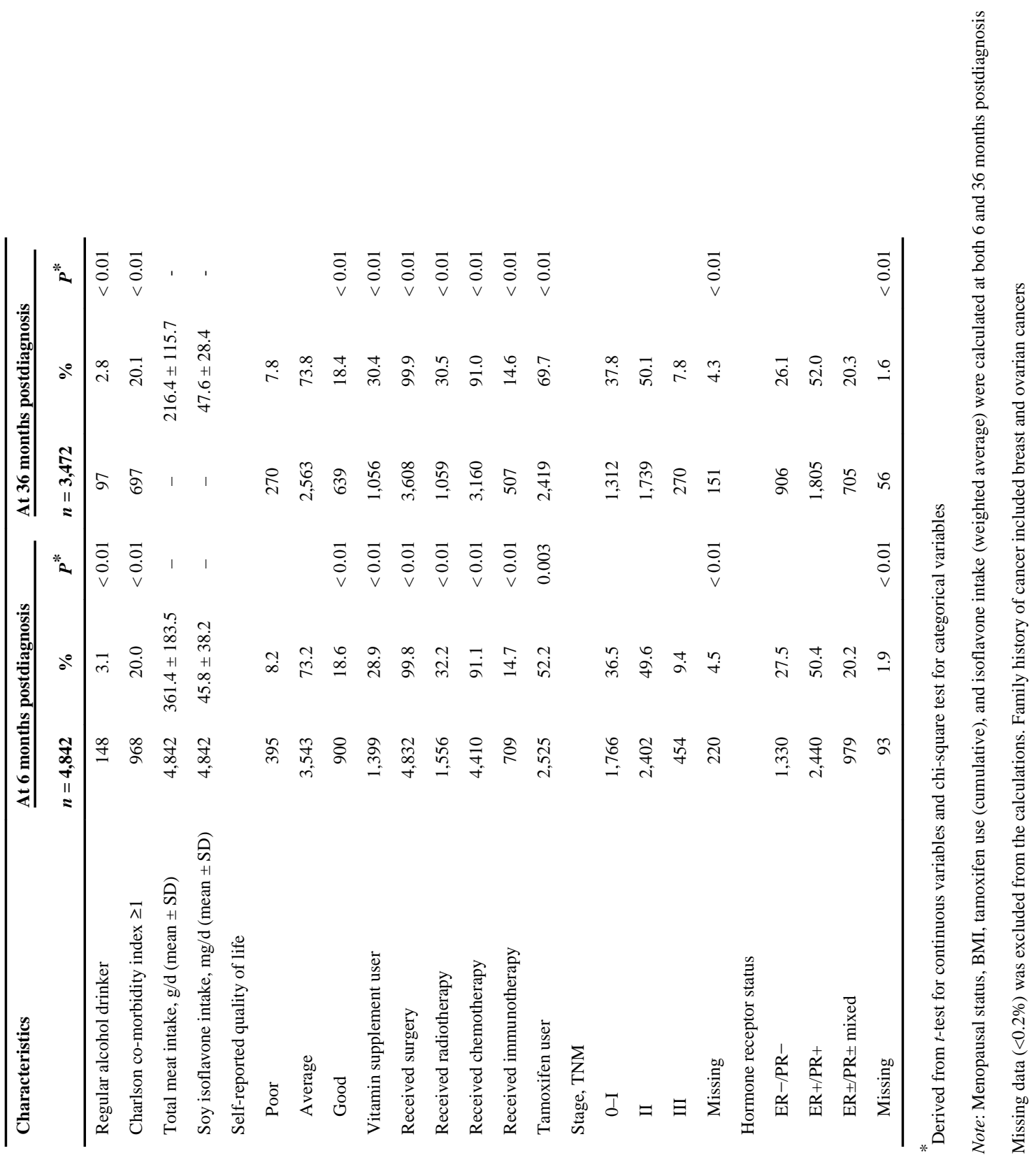




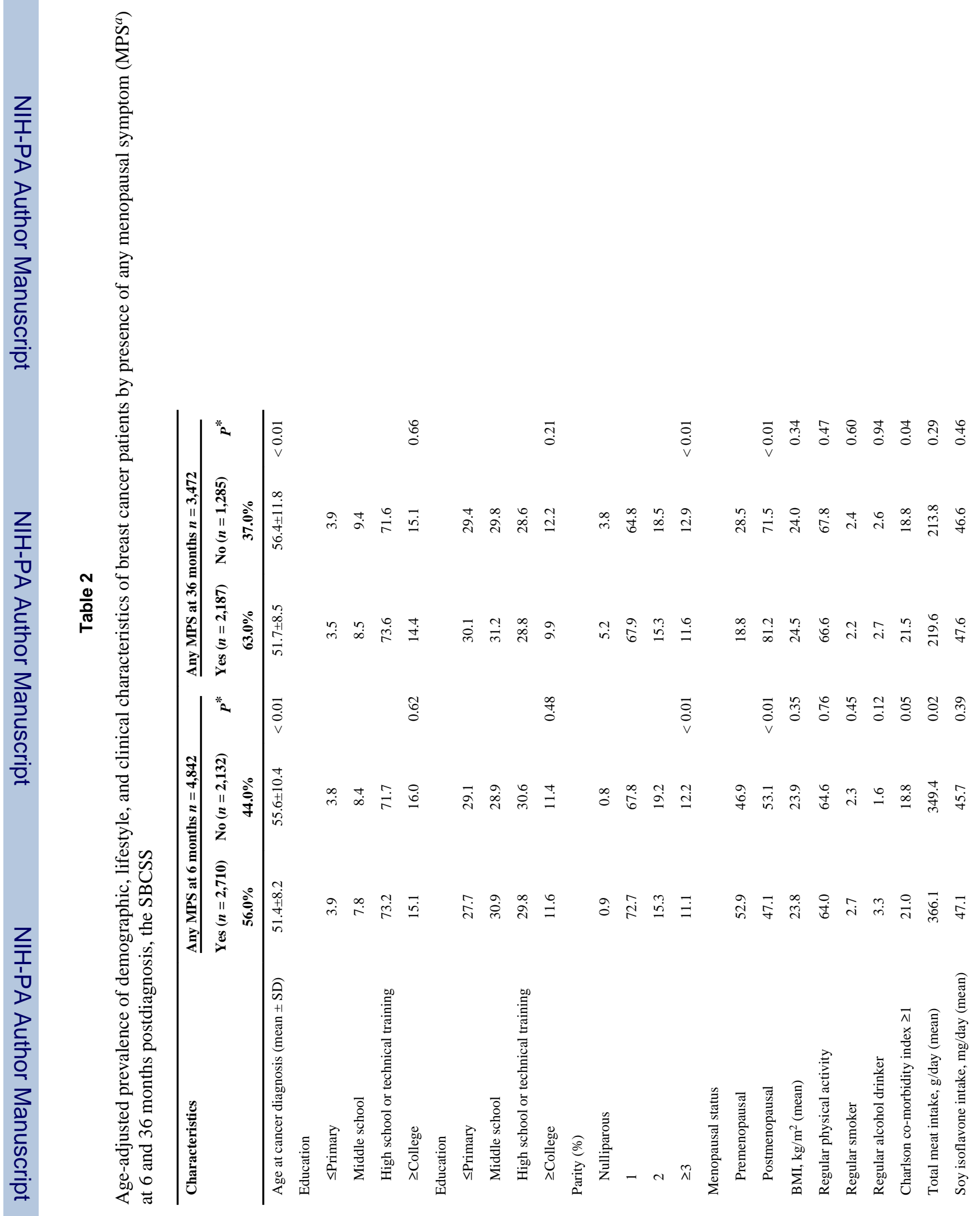




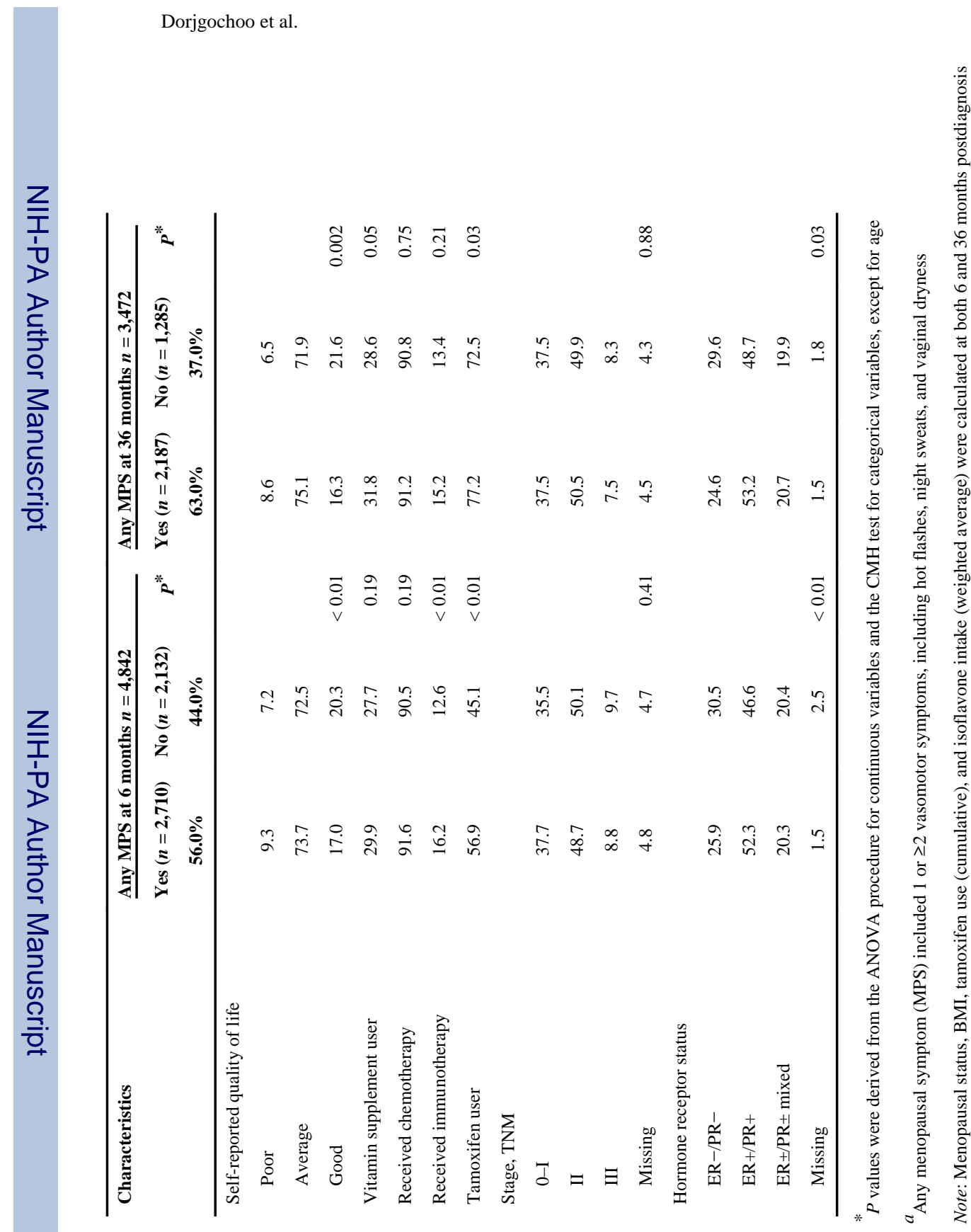

Page 13

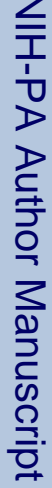

Breast Cancer Res Treat. Author manuscript; available in PMC 2012 December 1. 
ป

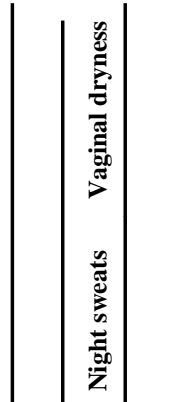

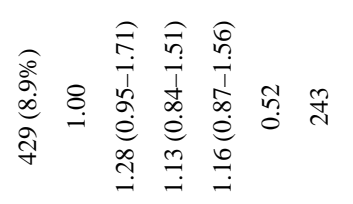

తิ $\widehat{\circ}$

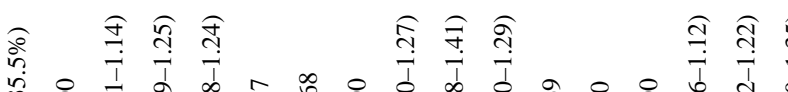

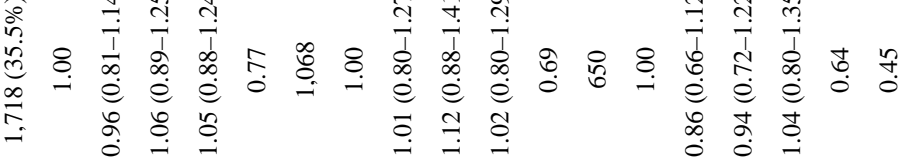

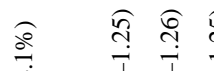

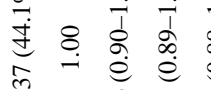

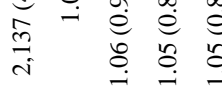

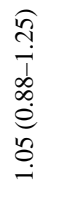

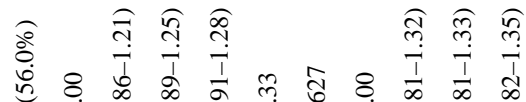

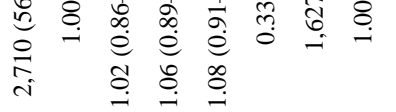

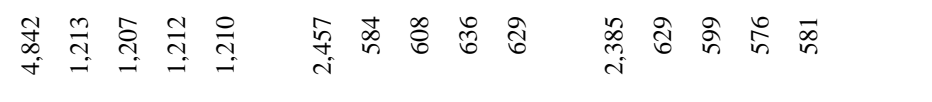

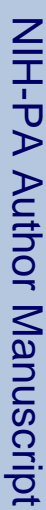

$\stackrel{\mathscr{\Sigma}}{\Sigma}$

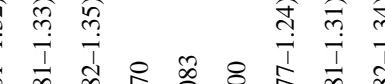

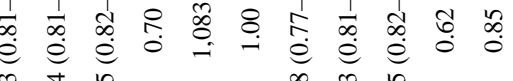

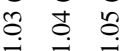

芴

ฮั

突

.

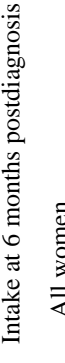

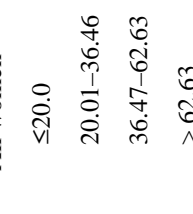

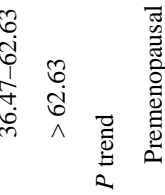

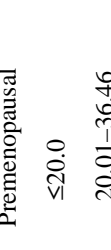

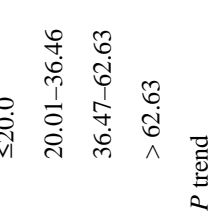

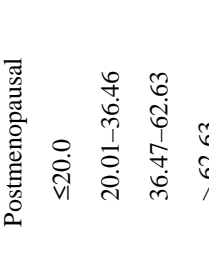

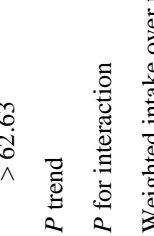

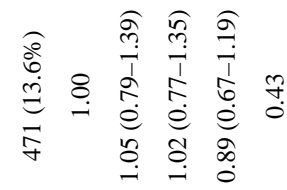

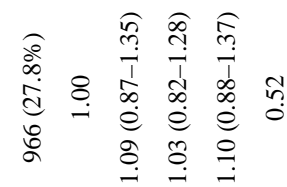

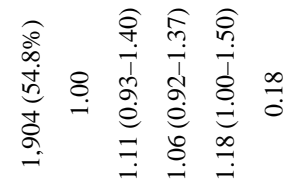

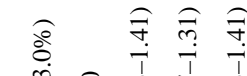

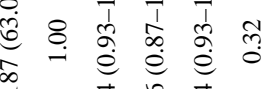

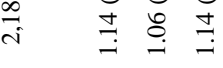

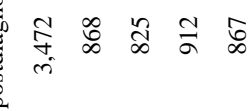
Breast Cancer Res Treat. Author manuscript; available in PMC 2012 December 1. 


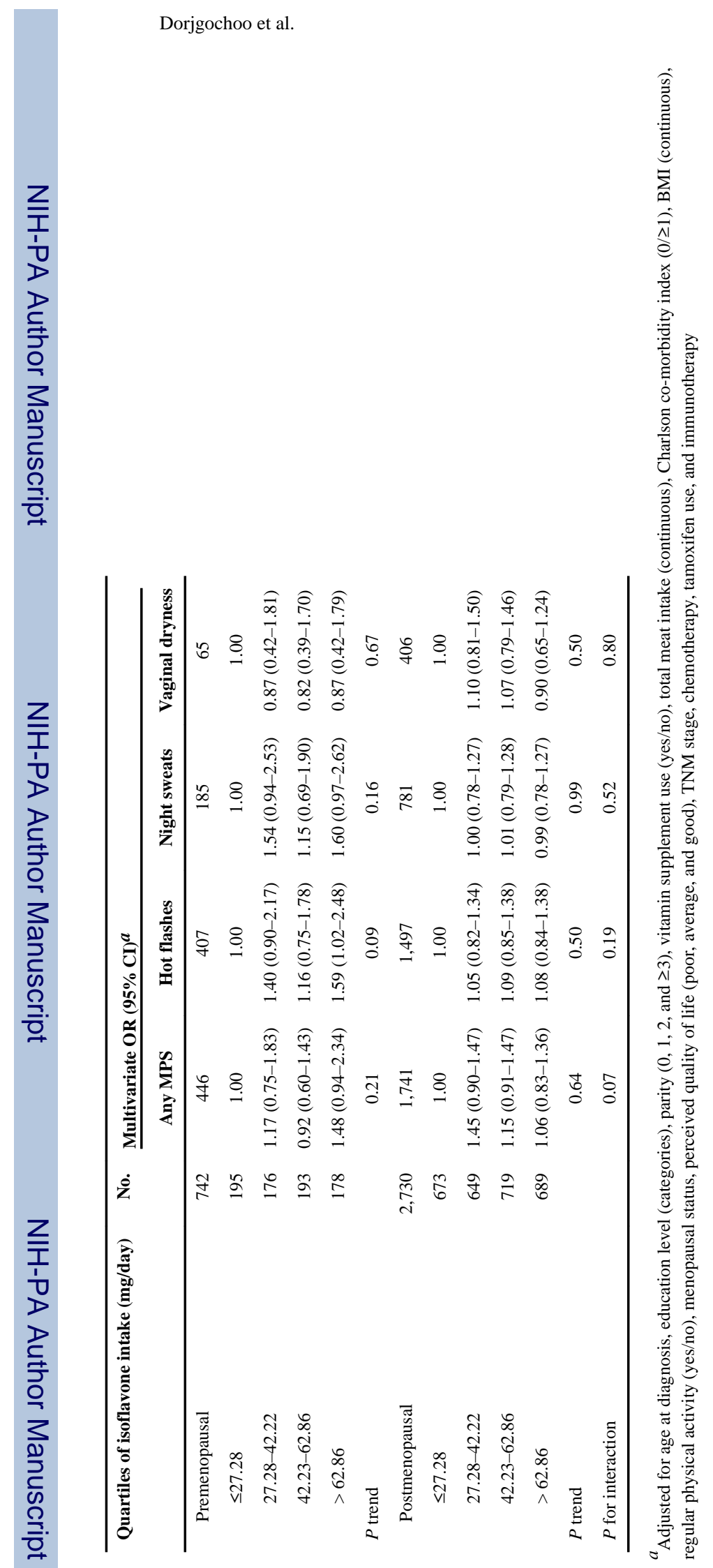

Breast Cancer Res Treat. Author manuscript; available in PMC 2012 December 1. 


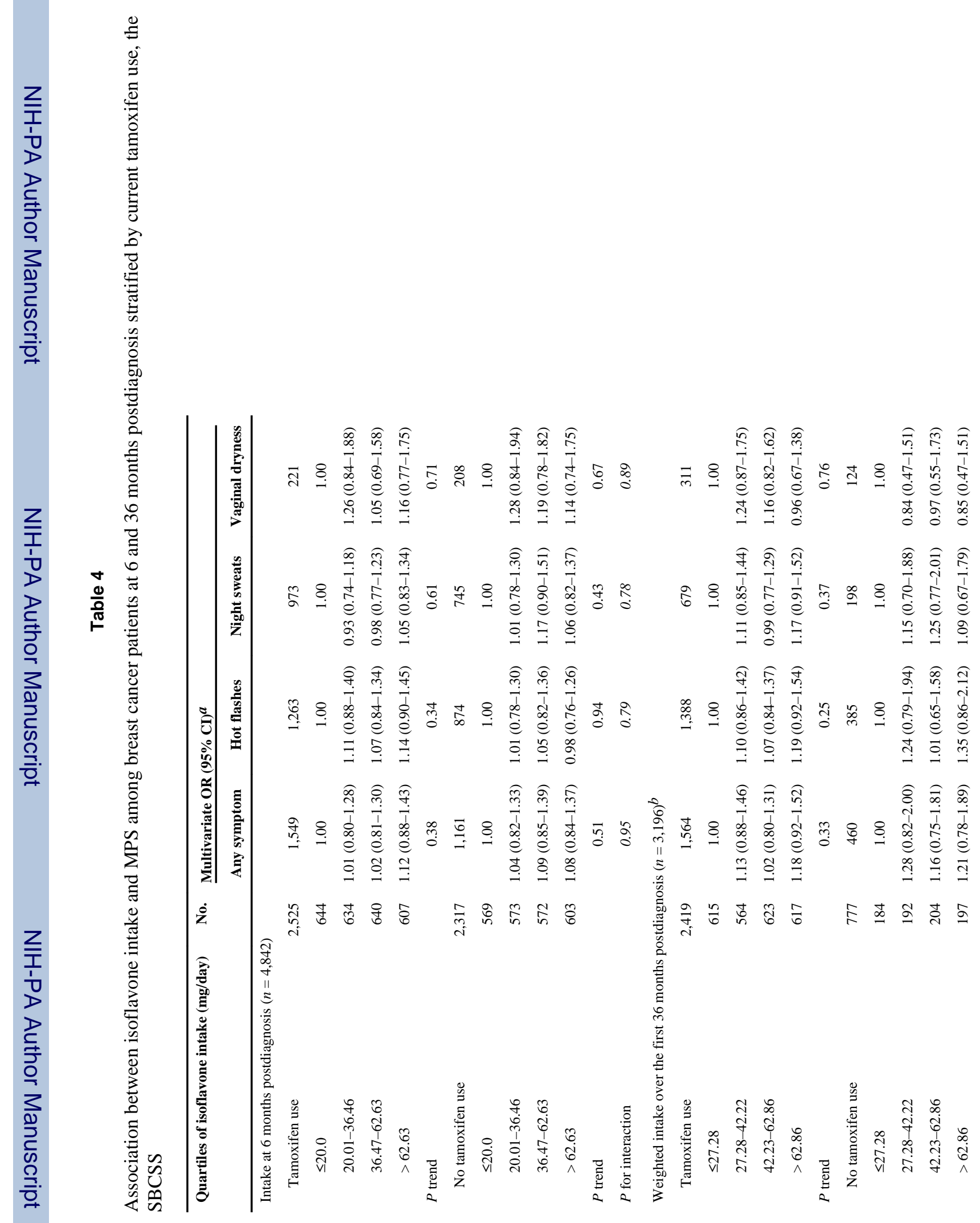




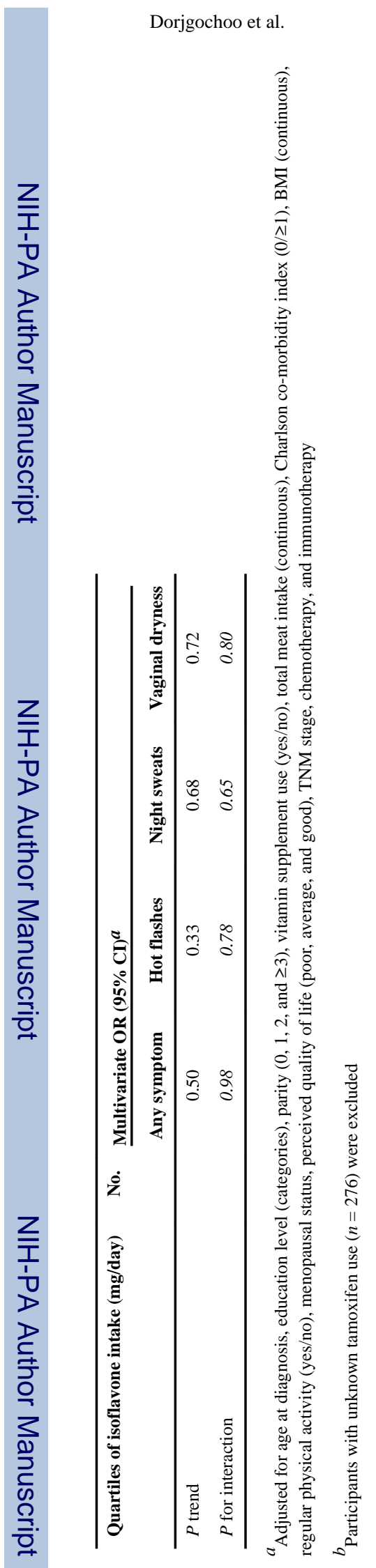

Breast Cancer Res Treat. Author manuscript; available in PMC 2012 December 1. 


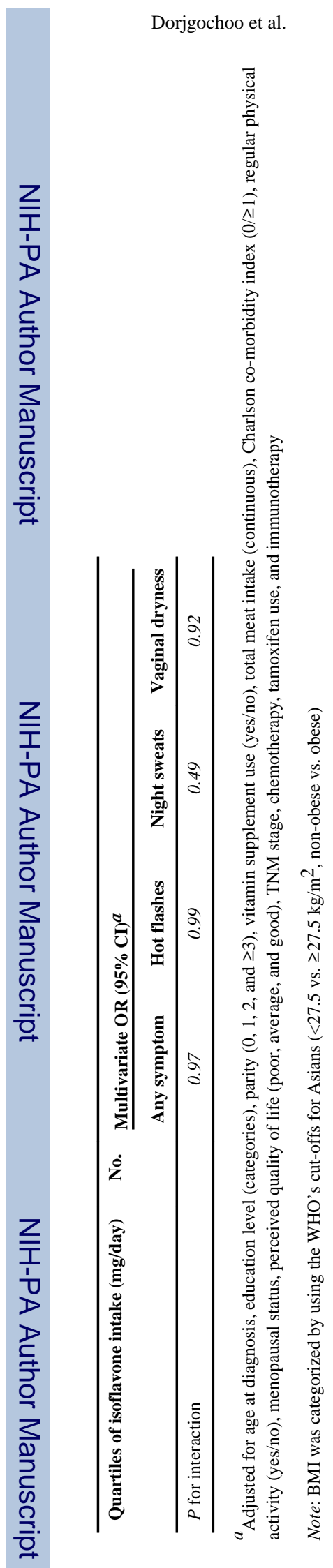

Breast Cancer Res Treat. Author manuscript; available in PMC 2012 December 1. 